\title{
PERIODIC TRAVELLING WAVE SELECTION BY DIRICHLET BOUNDARY CONDITIONS IN OSCILLATORY REACTION-DIFFUSION SYSTEMS*
}

\author{
JONATHAN A. SHERRATT ${ }^{\dagger}$
}

\begin{abstract}
Periodic travelling waves are a fundamental solution form in oscillatory reactiondiffusion equations. Here I discuss the generation of periodic travelling waves in a reaction-diffusion system of the generic $\lambda-\omega$ form. I present numerical results suggesting that when this system is solved on a semi-infinite domain subject to Dirichlet boundary conditions in which the variables are fixed at zero, periodic travelling waves develop in the domain. The amplitude and speed of these waves are independent of the initial conditions, which I generate randomly in numerical simulations. Using a combination of numerical and analytical methods, I investigate the mechanism of periodic travelling wave selection. By looking for an appropriate similarity solution, I reduce the problem to an ODE system. Using this, I derive a formula for the selected speed and amplitude as a function of parameters. Finally, I discuss applications of this work to ecology.
\end{abstract}

Key words. periodic waves, wavetrains, reaction-diffusion, oscillatory systems

AMS subject classification. 35K57

DOI. $10.1137 /$ S0036139902392483

1. Introduction. Periodic travelling waves (PTWs) are a fundamental solution form in oscillatory reaction-diffusion equations, by which I mean reaction-diffusion systems whose kinetics have a stable limit cycle. PTWs are the one-dimensional analogue of spiral waves and target patterns, and underlie many observed behaviors in biology and chemistry (Bjørnstad, Ims, and Lambin (1999); Scott et al. (2000)). In 1973, Kopell and Howard published their seminal paper, which showed that a reaction-diffusion system develops a one-parameter family of PTWs as its kinetics pass through a Hopf bifurcation. Wave speed or amplitude are convenient parameters for this family, and an oscillatory reaction-diffusion equation has a PTW solution for any speed above a critical minimum value and for any amplitude below that of the limit cycle in the kinetics. Building on Kopell and Howard's work, periodic travelling waves were studied extensively in the 1970s and 1980s. This work focussed primarily on the existence and stability of the solutions. For instance, Maginu (1981) showed that PTWs of sufficiently high speed are stable in general systems, and Ermentrout (1981) demonstrated stable small amplitude waves in a particular reaction-diffusion system. More recent work includes nonlinear stability analysis (Kapitula (1994)), the application of symmetry methods (Romero, Gandarias, and Medina (2000)), and the generation of PTWs behind invasive fronts (Sherratt (1994a,b); Sneyd and Sherratt (1997); Ermentrout, Chen, and Chen (1997); Petrovskii and Malchow (1999), (2000); Ashwin et al. (2002)).

The simplest behavior of an oscillatory system is a spatially uniform oscillation. In many cases, this solution is stable on an infinite, spatially homogeneous domain: for instance, in reaction-diffusion systems, stability is guaranteed when the diffusion coefficients are sufficiently similar (Kopell and Howard (1973)). However, spatially

\footnotetext{
*Received by the editors May 20, 2002; accepted for publication (in revised form) January 31, 2003; published electronically June 12, 2003. This research was supported in part by an EPSRC Advanced Research Fellowship.

http://www.siam.org/journals/siap/63-5/39248.html

${ }^{\dagger}$ Centre for Theoretical Modelling in Medicine, Department of Mathematics, Heriot-Watt University, Edinburgh EH14 4AS, UK (jas@ma.hw.ac.uk).
} 
uniform oscillations are incompatible with spatial heterogeneities, which can arise via spatially varying parameter values or via conditions imposed on finite boundaries. Such situations provide a potential mechanism for the generation of PTWs in oscillatory chemical or biological systems. For instance, it is well known that in experiments with the oscillatory Belousov-Zhabotinskii chemical reaction, small impurities such as dust particles force target patterns or spiral waves rather than homogeneous oscillations (Nagashima (1991), Winfree (2001)). Mathematically, the effects of such heterogeneities have been studied most fully for systems of discrete coupled oscillators. In particular, the work of Ermentrout, Kopell, and colleagues gives a detailed account of the response of chains of weakly coupled oscillators to both boundary- and parameter-based heterogeneities (Ermentrout and Kopell (1984), (1986); Kopell, Ermentrout, and Williams (1991); Ren and Ermentrout (1998)). In oscillatory reactiondiffusion systems, there has been some study of periodic wave generation by spatial inhomogeneities in the domain (Hagan (1981a); Kopell (1981); Kay and Sherratt (2000)). However, heterogeneities imposed at the edges of a domain have received little attention, despite early work by Auchmuty and Nicolis (1976), who developed series solutions for the Brusselator model close to Hopf bifurcation on a finite domain with Neumann and Dirichlet end conditions.

In the present paper, I study the generation of PTWs by particular Dirichlet conditions at one edge of a semi-infinite domain. In section 2, I introduce this behavior with the results of numerical simulations. In section 3, I show that solutions of the observed form satisfy an ODE system with one free parameter, which corresponds to the temporal frequency of the oscillations. I then present a combination of analytical and numerical results suggesting that this ODE system has a solution satisfying appropriate end conditions for a countably infinite set of values of this parameter. In section 5, I discuss the hypothesis that in only one of these solutions does the amplitude vary monotonically in space, and that this determines the stability of the solutions. In section 6 , I use a similarity solution to derive a formula for the speed and amplitude of the observed periodic wave. Finally, in section 7, I discuss extensions to two space dimensions and applications of the results.

2. Numerical simulations of PTW generation. All of the work in this paper involves the following oscillatory reaction-diffusion system:

$$
\begin{aligned}
& \frac{\partial u}{\partial t}=\nabla^{2} u+\left(1-r^{2}\right) u-\left(\omega_{0}-\omega_{1} r^{2}\right) v \\
& \frac{\partial v}{\partial t}=\nabla^{2} v+\left(\omega_{0}-\omega_{1} r^{2}\right) u+\left(1-r^{2}\right) v
\end{aligned}
$$

where $r=\sqrt{u^{2}+v^{2}}$. This belongs to the " $\lambda-\omega$ " class of equations introduced by Kopell and Howard (1973). The kinetics in (2.1) are the normal form of any oscillatory kinetics close to a supercritical Hopf bifurcation, and, as such, (2.1) is the natural system for studying generic behavior in systems in which each variable has the same diffusion coefficient. This system is often seen with $\left(1-r^{2}\right)$ replaced by $\left(\lambda_{0}-\lambda_{1} r^{2}\right)$, but the coefficients $\lambda_{0}$ and $\lambda_{1}$ can easily be removed by rescaling. All of the work in sections $2-6$ is in one space dimension; in section 7 , two-dimensional behavior is discussed briefly.

The kinetics of (2.1) have an unstable equilibrium at $u=v=0$ and a stable circular limit cycle centered at this equilibrium, of radius 1 . Standard theory, due 
originally to Kopell and Howard (1973), shows that the family of PTWs is given by

$$
\begin{aligned}
& u=r^{*} \cos \left[\theta_{0} \pm \sqrt{1-r^{* 2}} x+\left(\omega_{0}-\omega_{1} r^{* 2}\right) t\right] \\
& v=r^{*} \sin \left[\theta_{0} \pm \sqrt{1-r^{* 2}} x+\left(\omega_{0}-\omega_{1} r^{* 2}\right) t\right]
\end{aligned}
$$

where $r^{*}$ parameterizes the family and $\theta_{0}$ is an arbitrary constant. The wave is stable as a solution of $(2.1)$, provided that

$$
r^{*}>r_{s t a b} \equiv\left(\frac{2+2 \omega_{1}^{2}}{3+2 \omega_{1}^{2}}\right)^{1 / 2}
$$

(Kopell and Howard (1973)). In many situations, it is convenient to rewrite system (2.1) using $r$ and $\theta=\tan ^{-1}(v / u)$, which are polar coordinates in the $u-v$ plane. In one space dimension, this gives

$$
\begin{aligned}
& r_{t}=r_{x x}-r \theta_{x}^{2}+r\left(1-r^{2}\right), \\
& \theta_{t}=\theta_{x x}+\frac{2 r_{x} \theta_{x}}{r}+\omega_{0}-\omega_{1} r^{2} .
\end{aligned}
$$

Here and throughout the paper, the suffixes ${ }_{x}$ and $t$ denote derivatives. The PTW solutions (2.2) are of course given in terms of $r$ and $\theta$ by

$$
r=r^{*}, \quad \theta=\theta_{0} \pm \sqrt{1-r^{* 2}} x+\left(\omega_{0}-\omega_{1} r^{* 2}\right) t .
$$

In fact, it is easy to show that any solution with $r$ constant and $<1$ is a PTW.

The starting point of my work is the following very simple situation. I consider (2.1) on a semi-infinite domain $x>0$, say, with the boundary condition $u=v=0$ at $x=0$. Numerically this can be reproduced by solving on the finite domain $0<$ $x<X_{\infty}$, with $X_{\infty}$ large and with $u_{x}=v_{x}=0$ at $x=X_{\infty}$. I consider the solution that develops from random initial conditions, by which I mean that I use a random number generator to calculate $u$ and $v$ values, between \pm 1 , at points with an equal spacing of about $\Delta x=5$ throughout the domain, and then join these random values by straight lines to give the initial condition.

For a wide range of values of the parameters $\omega_{0}$ and $\omega_{1}$, the numerical solutions of this problem show the same behavior (Figure 1). The solution changes rapidly from the random initial conditions to spatially uniform oscillations everywhere away from the $x=0$ boundary. A transition wave then develops, which has homogeneous oscillations ahead of it and a PTW behind; this PTW is the long term solution form away from the $x=0$ boundary. The development and persistence of PTWs in these solutions depends intrinsically on the boundary condition at $x=0$. For example, if the boundary condition is switched to zero flux $\left(u_{x}=v_{x}=0\right.$ at $\left.x=0\right)$, the PTWs disappear, to be replaced by spatially uniform oscillations (see Kay and Sherratt (1999)). Moreover, the speed/amplitude of the PTWs is independent of the seed in the random number generator used for the initial conditions. This suggests that the Dirichlet boundary condition robustly selects a particular member of the PTW family. The basic goal of the paper is to investigate the details of this selection process.

Before I begin analytical investigation of the solution shown in Figure 1, I mention one final and important result from the numerical simulations. The behavior illustrated in Figure 1 applies when $\left|\omega_{1}\right|$ is relatively small. For larger $\left|\omega_{1}\right|$, the long 


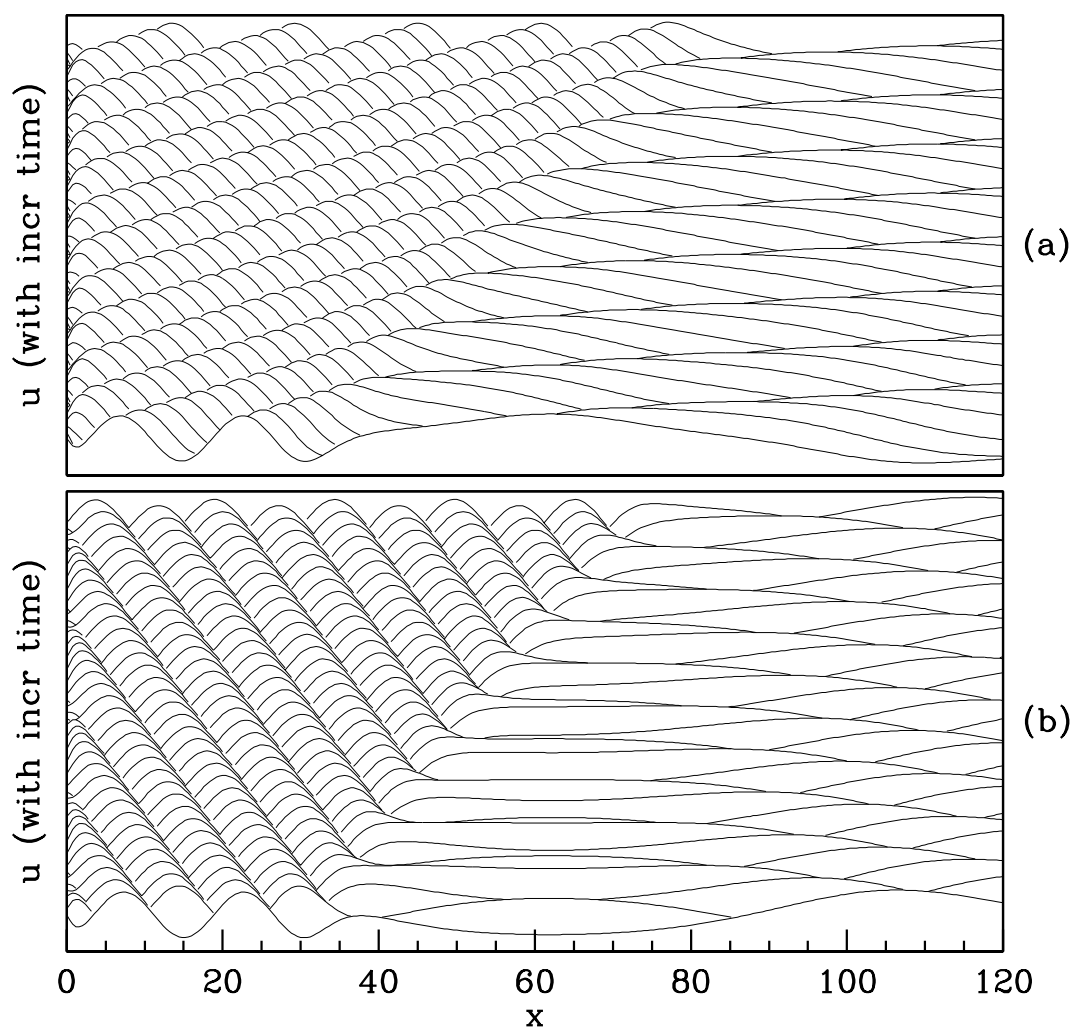

FIG. 1. Solutions of (2.1) with boundary conditions $u=v=0$ at $x=0$, and $u_{x}=v_{x}=0$ at $x=400$; only part of the solution is plotted. A transition front moves across the domain, behind which PTWs develop, moving in the positive $x$-direction in (a), and the negative $x$-direction in (b). The solutions are space-time plots, with $u$ plotted at equally spaced times between $t=100$ and $t=200$ (time increasing up the page). The solutions for $v$ are qualitatively similar. Initial conditions $(t=0)$ are generated randomly as described in the main text. The parameter values are $\omega_{1}=1.0$ and (a) $\omega_{0}=1.5$, (b) $\omega_{0}=-1.3$. The equations were solved numerically using a semi-implicit Crank-Nicolson method.

term behavior consists not of PTWs, but of irregular spatiotemporal oscillations (Figure 2). Later in the paper, I will show that this behavior arises through the same basic mechanism and occurs when the PTW that is selected by the boundary conditions has an amplitude below $r_{s t a b}$, defined in (2.3), so that the selected PTW is unstable as a solution of the PDEs.

3. Reduction to an ODE system. The solutions shown in Figure 1 are illustrated more clearly by plotting $r$ and $\theta_{x}$ rather than $u$ and $v$ (Figure 3). The solution changes rapidly from the initial conditions, until $r \approx 1$ and $\theta_{x} \approx 0$ everywhere away from the $x=0$ boundary, corresponding to spatially homogeneous oscillations in $u$ and $v$. A transition wave front in $r$ and $\theta_{x}$ then develops, moving in the positive $x$-direction. Ahead of this front, $r \rightarrow 1$ and $\theta_{x} \rightarrow 0$; behind it, $r$ and $\theta_{x}$ have constant values, $r_{p t w}$ and $\psi_{p t w}$ say, corresponding to the PTW. Numerical results indicate that this transition front moves with constant shape and speed, suggesting that one look 


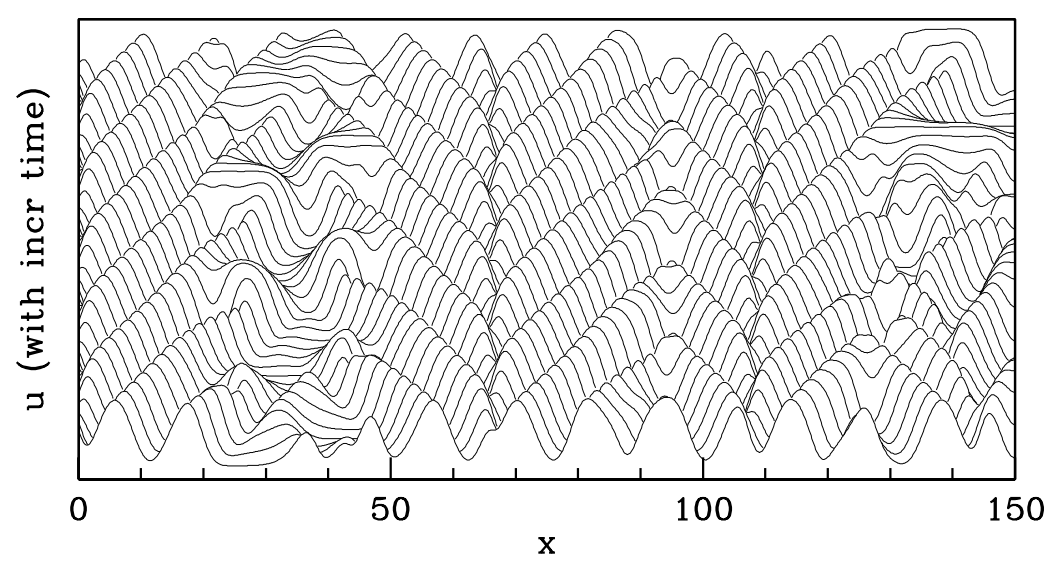

FIG. 2. Long-term solution of (2.1) for which irregular spatiotemporal oscillations develop. The boundary conditions are $u=v=0$ at $x=0$, and $u_{x}=v_{x}=0$ at $x=500$; only part of the solution is plotted. Note that a band of PTWs is visible close to the $x=0$ boundary. The solution is a spacetime plot, with $u$ plotted at equally spaced times between $t=1900$ and $t=2000$ (time increasing up the page). The solution for $v$ is qualitatively similar. Initial conditions $(t=0)$ are generated randomly as described in the main text. The parameter values are $\omega_{0}=1.5$ and $\omega_{1}=1.65$. The equations were solved numerically using a semi-implicit Crank-Nicolson method.

for solutions of (2.1) with the form

$$
r(x, t)=\hat{r}(x-s t) \quad \text { and } \quad \theta_{x}(x, t)=\hat{\psi}(x-s t) \Rightarrow \theta(x, t)=\int^{z=x-s t} \hat{\psi}(z) d z+f(t) .
$$

Here $s>0$ is the front speed, and $f(t)$ is an arbitrary function of time that enters as a constant of integration. Substituting these solution forms into (2.4) gives

$$
\begin{aligned}
\hat{r}^{\prime \prime}+s \hat{r}^{\prime}+\hat{r}\left(1-\hat{r}^{2}-\hat{\psi}^{2}\right) & =0, \\
\hat{\psi}^{\prime}+s \hat{\psi}+\omega_{0}-\omega_{1} \hat{r}^{2}+2 \hat{\psi} \hat{r}^{\prime} / \hat{r} & =f^{\prime}(t) .
\end{aligned}
$$

Thus $f^{\prime}(t)$ must be a constant, independent of $t$. Moreover, since $\hat{r} \rightarrow 1$ and $\hat{\psi} \rightarrow 0$ as $x-s t \rightarrow \infty$, this constant value must be $\omega_{0}-\omega_{1}$. Substituting $\hat{r}=r_{p t w}$ and $\hat{\psi}=\psi_{p t w}$ (values at $x-s t=-\infty$ ) gives solutions for $r_{p t w}$ and $\psi_{p t w}$ in terms of $s$ :

$$
r_{p t w}=\sqrt{1-\frac{s^{2}}{\omega_{1}^{2}}}, \quad \psi_{p t w}=-\frac{s}{\omega_{1}} .
$$

Unfortunately, these formulae cannot be used to obtain the values of $r_{p t w}$ and $\psi_{p t w}$, since the front speed $s$ is an unknown. However, they do provide one key piece of information: $\psi_{p t w}$ has the sign opposite to that of $\omega_{1}$, since $s$ must be positive. This will be required in what follows.

Having established the sign of $\psi_{p t w}$, I now move on to consider the large time form of the solution for $r$ and $\theta_{x}$. Numerical simulations suggest that this is an equilibrium, which I denote by $r(x, t)=R(x)$ and $\theta_{x}(x, t)=\Psi(x)$. Hence $\theta=\int^{x} \Psi(\bar{x}) d \bar{x}+g(t)$, where $g(t)$ is a constant of integration. Substituting these solution forms into (2.4) implies that $g^{\prime}(t)$ must be a constant, which it is convenient to take as $\omega_{0}-k$, where 


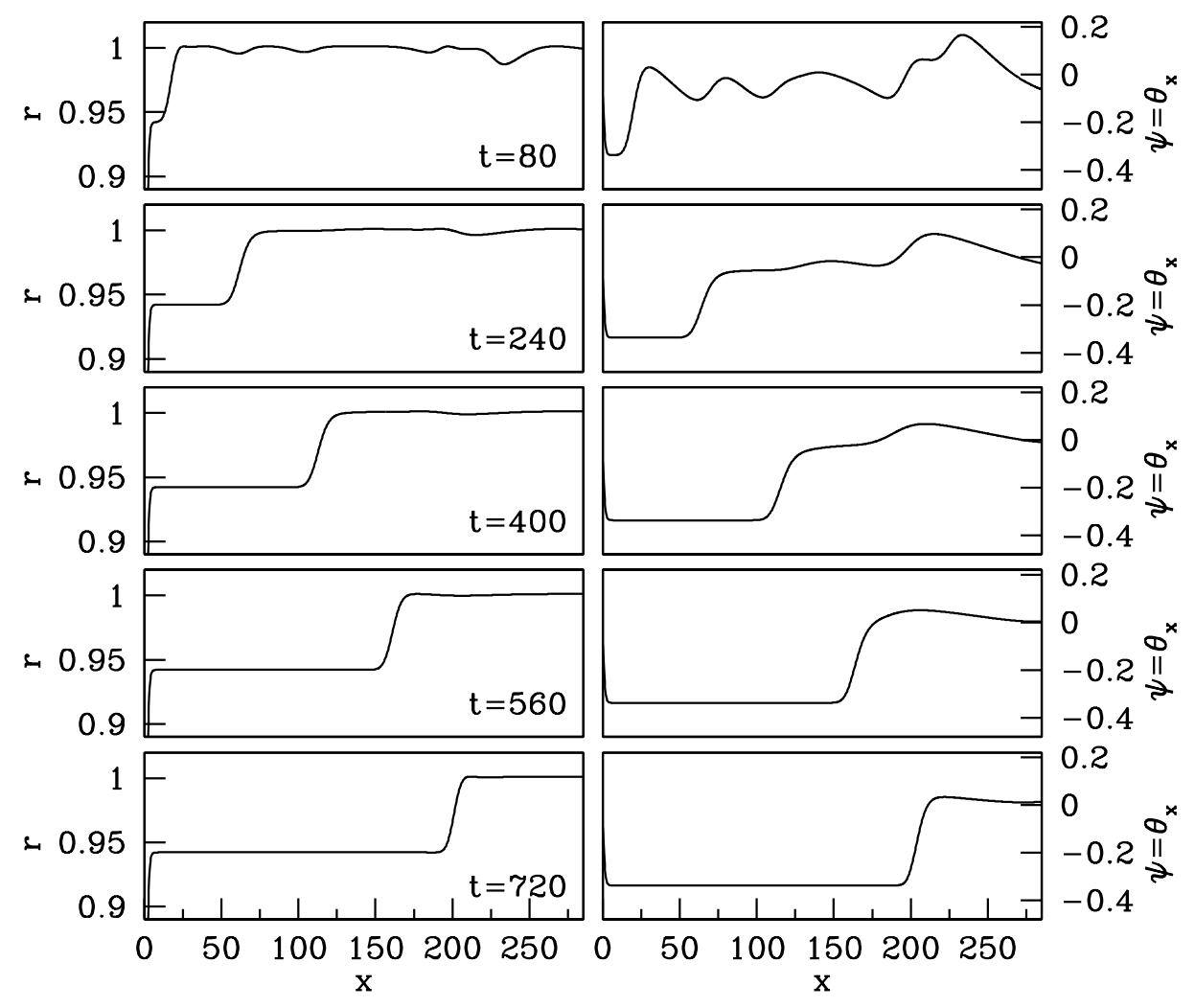

FIG. 3. Time evolution of the solution of (2.1) subject to $u=v=0$ at $x=0$. I solve on $0<x<400$ with $u_{x}=v_{x}=0$ at $x=400$, to approximate a semi-infinite domain. The randomly generated initial condition rapidly evolves to $u, v \approx 1$. A transition wave front then develops, moving in the positive $x$-direction. Ahead of the front, $r=1$ and $\psi=0$, while behind it, $r$ and $\psi$ have values that are constants corresponding to a periodic travelling wave. The parameter values are $\omega_{0}=0.3$ and $\omega_{1}=0.8$. The equations were solved numerically using a semi-implicit Crank-Nicolson method.

$k$ is arbitrary and of either sign. The substitution also gives the following equations for $R$ and $\Psi$ :

$$
\begin{aligned}
R_{x x}+R\left(1-R^{2}-\Psi^{2}\right) & =0, \\
\Psi_{x}+\frac{2 \Psi R_{x}}{R}+k-\omega_{1} R^{2} & =0 .
\end{aligned}
$$

The boundary condition $u=v=0$ implies that $R=0$ at $x=0$, and I am looking for solutions for which $R$ and $\Psi$ tend to constant values, denoted $r_{p t w}$ and $\psi_{p t w}$, as $x \rightarrow \infty$, with the sign of $\psi_{p t w}$ opposite to that of $\omega_{1}$. In a solution of this form, the values of $r_{p t w}$ and $\psi_{p t w}$ will be related to $k$ and $\omega_{1}$ by

$$
r_{p t w}=\sqrt{\frac{k}{\omega_{1}}}, \quad \psi_{p t w}=-\operatorname{sign}\left(\omega_{1}\right) \sqrt{1-\frac{k}{\omega_{1}}} .
$$

These are given simply by substituting the constant values into (3.3) and using the result that $\psi_{p t w}$ and $\omega_{1}$ have opposite signs; $k$ is related to the speed $s$ introduced above by $k=\omega_{1}-s^{2} / \omega_{1}$. Note that $k$ must have the same sign as $\omega_{1}$. 
It is convenient to rescale (3.3) as follows,

$$
\begin{gathered}
\phi=R\left(\frac{\omega_{1}}{k}\right)^{1 / 2}, \quad w=R_{x}\left(\frac{\omega_{1}-k}{k}\right)^{1 / 2} \cdot \frac{\operatorname{sign}\left(\omega_{1}\right)}{k}, \\
\Gamma=-\frac{\Psi}{R}\left(\frac{k}{\omega_{1}-k}\right)^{1 / 2} \operatorname{sign}\left(\omega_{1}\right), \quad z=x\left(\frac{\omega_{1}}{\omega_{1}-k}\right)^{1 / 2} \cdot k \cdot \operatorname{sign}\left(\omega_{1}\right),
\end{gathered}
$$

which gives

$$
\begin{aligned}
\phi_{z} & =w, \\
w_{z} & =\frac{-\alpha}{k^{2}} \phi\left[1-\phi^{2}-\alpha \phi^{2}\left(\Gamma^{2}-1\right)\right], \\
\Gamma_{z} & =\frac{1-3 w \Gamma-\phi^{2}}{\phi}
\end{aligned}
$$

where $\alpha=1-k / \omega_{1}$, so that $0 \leq \alpha \leq 1$. In terms of these new variables, the required end conditions are

$$
\phi=0 \text { at } z=0 \quad \text { and } \quad \phi=1, w=0, \Gamma=1 \text { at } z=\infty .
$$

Recall that the parameter $k$ in (3.5) is an arbitrary constant of integration, and the initial question to be studied is for which values of $k$ there are solutions of (3.5) satisfying these end conditions.

Numerical investigation of appropriate solutions to (3.5) is easiest if one integrates backwards in $z$ from $(1,0,1)$. Straightforward calculation of the eigenvalues at this equilibrium shows that there is a unique stable eigenvector, and one can calculate numerically both trajectories corresponding to this eigenvector. For given values of $k$ and $\omega_{1}$, there is a solution of (3.5) of the required form if $\phi$ becomes zero along one of these trajectories. Numerical investigation indicates that this occurs at a large but discrete set of values of $k$. As illustrated in Figure 4, these values of $k$ are widely separated when $|k|$ is just below $\left|\omega_{1}\right|$, and become closer together as $|k|$ approaches zero. (Recall that the sign of $k$ is determined by that of $\omega_{1}$.) Figure 5 illustrates how the critical values of $k$ vary with $\omega_{1}$.

4. Solution for small $\omega_{1}$. I have been unable to calculate in general the values of $k$ for which (3.5) has a solution of the required form. However for small $\left|\omega_{1}\right|$ the solutions, and thus the critical values of $k$, can be found using perturbation theory. Here I am exploiting the relative simplicity of (2.1) when $\omega_{1}=0$, a special case which has been used by a number of previous authors (for example, Kopell and Howard (1981)). Figure 6 illustrates the typical form of the solution for the largest few critical values of $|k|$ when $\left|\omega_{1}\right|$ is small. There is a characteristic solution form, with almost periodic oscillations in $\phi, w$, and $\Gamma$. To calculate the solutions, it is enough to investigate one cycle of these oscillations.

Numerical solutions suggest that when $\left|\omega_{1}\right|$ is small, $|k|$ is also small, with the ratio $A \equiv \alpha / k^{2}$ being $O(1)$ as $\left|\omega_{1}\right| \rightarrow 0$. Then $\alpha^{2} / k^{2}=A^{2} \omega_{1}^{2}+O\left(\omega_{1}^{4}\right)$, and thus the equations (3.5) have the form

$$
\begin{aligned}
\phi_{z z} & =-A \phi\left(1-\phi^{2}\right)+\epsilon A^{2} \phi^{3}\left(\Gamma^{2}-1\right), \\
\Gamma_{z} & =\frac{1-3 \phi_{z} \Gamma-\phi^{2}}{\phi}
\end{aligned}
$$




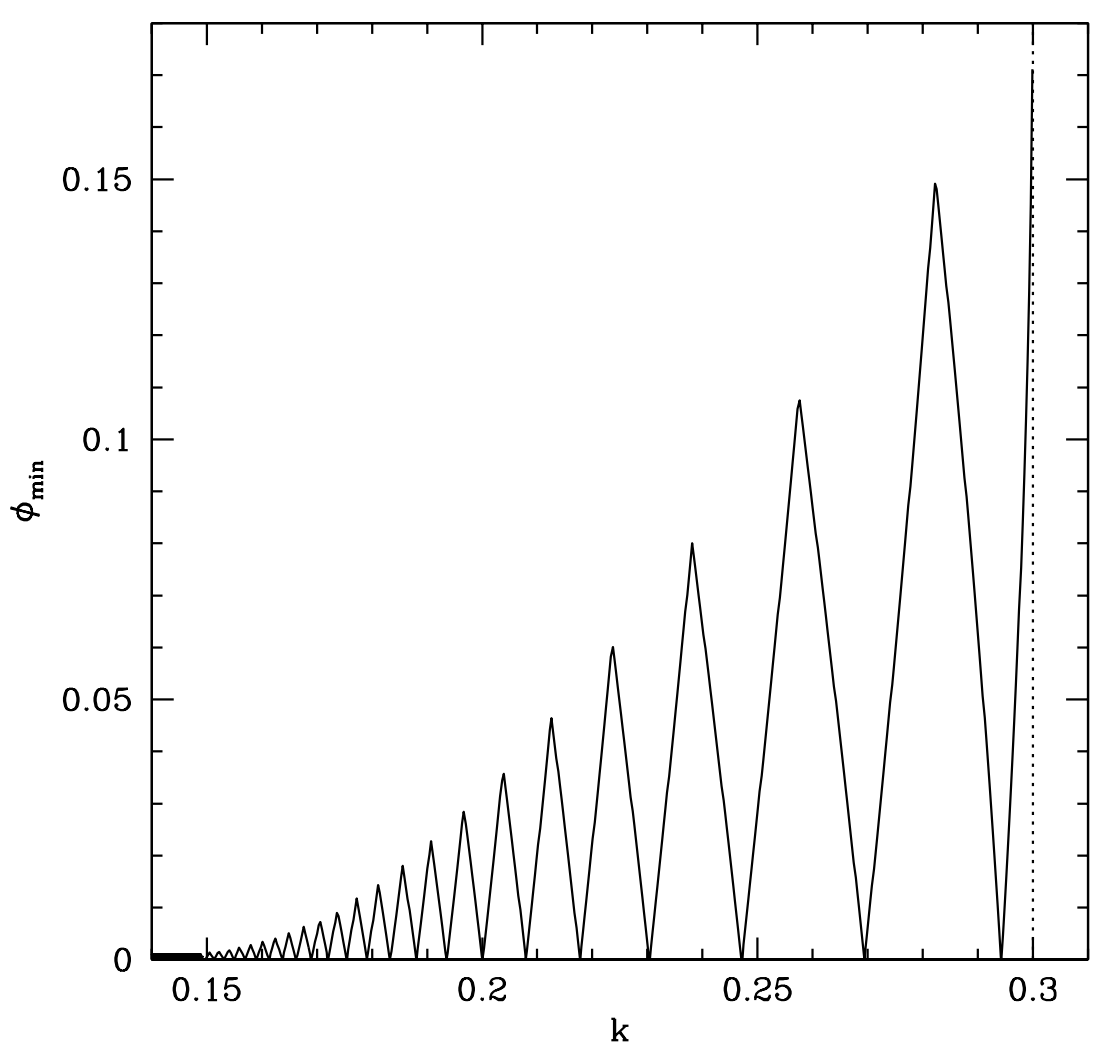

FIG. 4. A typical plot of the variation with $k$ of $\phi_{\text {min }}$, the minimum value of $\phi$ along the two solution trajectories passing through the point $\phi=1, w=0, \Gamma=1$. The variation has a "zigzag" form, with $\phi_{\text {min }}$ approaching zero at a series of discrete values of $k$. The case shown is for $\omega_{1}=0.3$, and the dotted line is $k=\omega_{1}$; the constant $k$ must lie between 0 and $\omega_{1}$. The value of $\phi_{\min }$ is calculated by solving (3.5) in the negative $z$-direction starting on the (unique) stable eigenvector at $(1,0,1)$. The solution is continued until $\phi>1$, keeping track of the minimum value of $\phi$. For each parameter set, this procedure must be followed twice, starting on either side of $(1,0,1)$ along the stable eigenvector.

where $\epsilon=\omega_{1}^{2}$. The appropriate solution structure for these equations when $\epsilon \ll 1$ is illustrated in Figure 7. I consider one cycle of the solution in three separate regions ac, with a fourth region a' corresponding to region a in the next cycle. The boundary between regions $\mathrm{a}$ and $\mathrm{b}$ is the position at which $\phi$ has its local maximum, while region $\mathrm{c}$ is a thin layer centered on the local minimum of $\phi$. No thin transition layer is required between regions a and $\mathrm{b}$, but the location $z=z_{1}$ of the interface may depend on $\epsilon$, as may the location $z=z_{2}$ of region $\mathrm{c}$, and these dependencies must be found as part of the solution. The position $z=z_{0}$ to the left of region a is arbitrary.

In region a, there is no rescaling, and the leading order solutions $\phi_{0}^{a}, \Gamma_{0}^{a}$ satisfy (4.1) with $\epsilon$ set to zero. The two equations decouple, giving

$$
\begin{aligned}
\frac{d^{2} \phi_{0}^{a}}{d z^{2}} & =-A \phi_{0}^{a}\left[1-\phi_{0}^{a 2}\right], \\
\frac{d \Gamma_{0}^{a}}{d z} & =\frac{1-3 \Gamma_{0}^{a} d \phi_{0}^{a} / d z-\phi_{0}^{a 2}}{\phi_{0}^{a}} .
\end{aligned}
$$




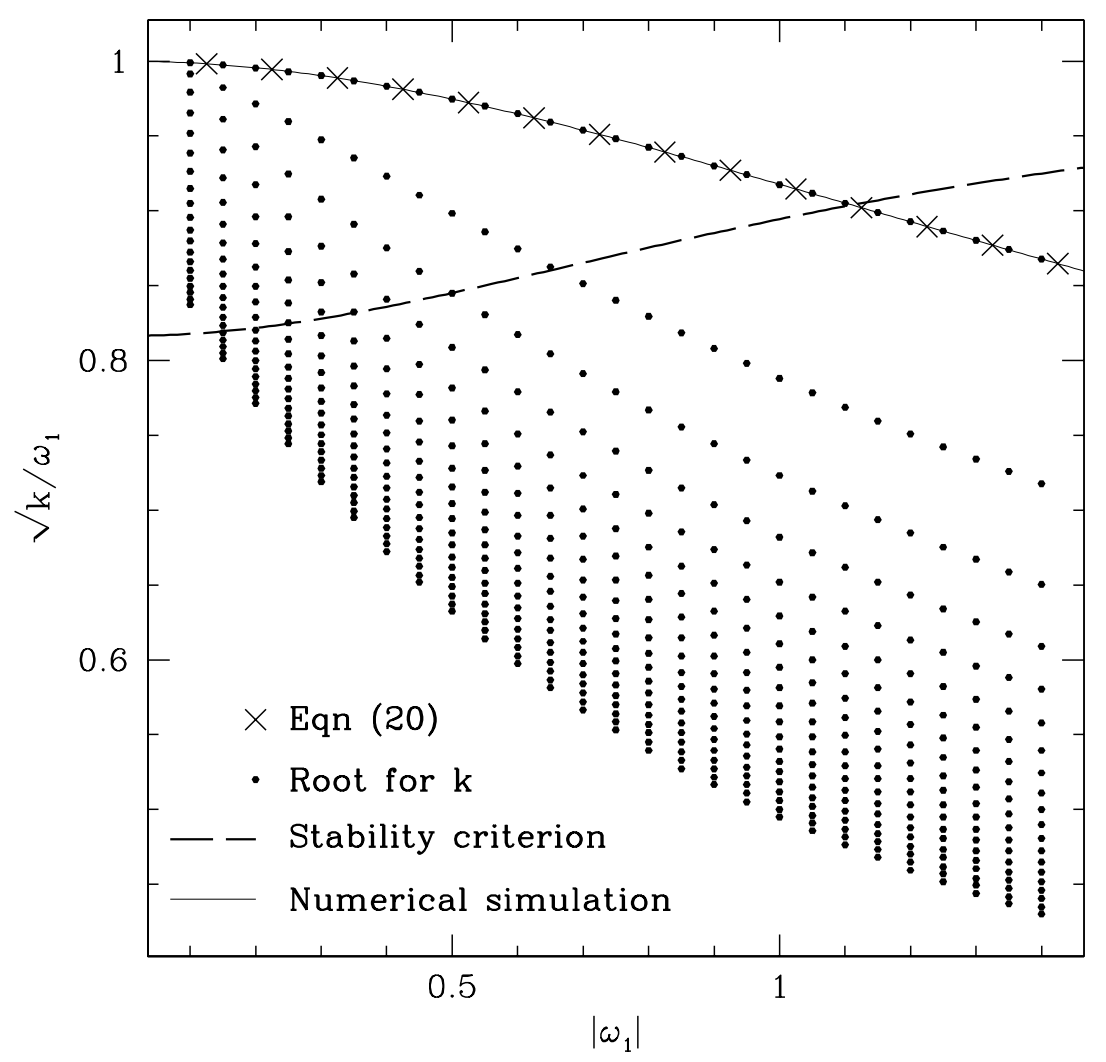

FIG. 5. A plot of the amplitude $\left(\sqrt{k / \omega_{1}}\right)$ of possible PTW solutions, corresponding to the critical values of $k$ for which (3.5) has a solution of the required form. For a series of values of $\omega_{1}$, I plot the amplitude corresponding to the largest 20 critical values of $k$, calculated numerically as discussed in the legend to Figure 4. Superimposed on the plot are the amplitude of PTWs predicted by numerical simulations of the PDEs (2.1), the theoretical prediction (6.2) of the PTW amplitude, and the curve determining PTW stability, which is given by (2.3). Note that this last curve does not refer to the stability of the solution of (3.5), (3.6), but simply to the PTW which this solution approaches as $z \rightarrow \infty$. Stability of this PTW is clearly a necessary but not sufficient condition for the stability of the solution of (3.5), (3.6).

Thus

$$
\left(\frac{d \phi_{0}^{a}}{d z}\right)^{2}=\frac{1}{2} A\left(\phi_{0}^{a}{ }^{2}-1\right)^{2}+C_{1},
$$

where $C_{1}$ is a constant of integration. Numerical solutions suggest that the local maxima in $\phi$ occur at $\phi=1+o(1)$ as $\epsilon \rightarrow 0$, and thus $C_{1}=0$. By construction, $\phi_{0}^{a}$ has positive slope, and thus further integration gives

$$
\begin{aligned}
\phi_{0}^{a} & =\tanh \left[\left(z-z_{0}\right) \sqrt{\frac{A}{2}}\right], \\
\Gamma_{0}^{a} & =\sqrt{\frac{2}{9 A}}+\frac{k_{1}}{\tanh ^{3}\left[\left(z-z_{0}\right) \sqrt{A / 2}\right]} .
\end{aligned}
$$

Here I am taking $\phi_{0}^{a}=0$ at $z=z_{0}$, since numerical solutions suggest that the minima of $\phi$ are $o(1)$ as $\epsilon \rightarrow 0 ; k_{1}$ is a constant of integration. 

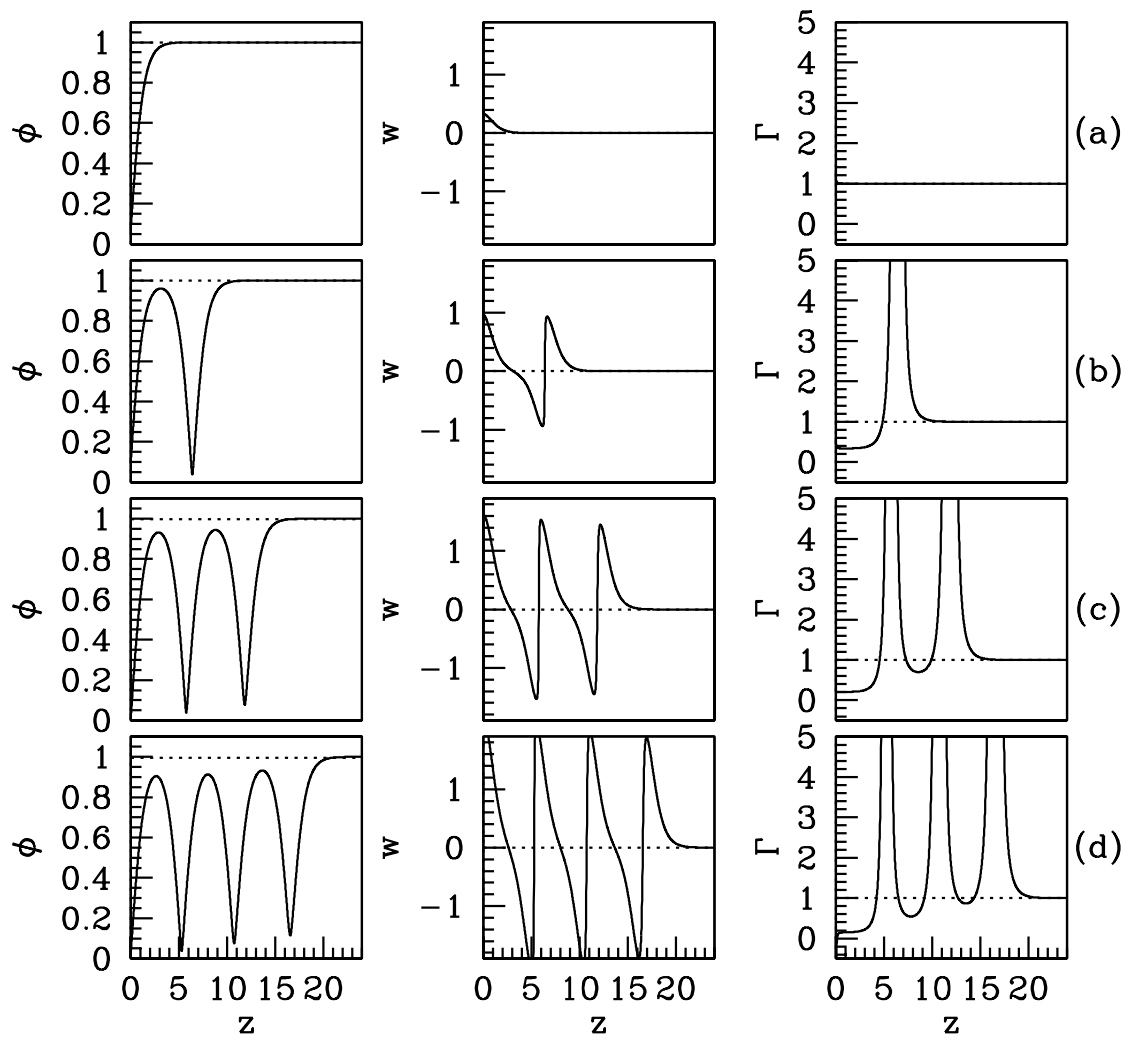

FIG. 6. Typical solutions of (3.5) for small $\left|\omega_{1}\right|$. I show the solution for the largest three critical values of $|k|$, illustrating the typical form of the solution. For the largest value of $|k|$, the solution is monotonic in $\phi$, and in successive solutions the variables cycle. The parameter values are $\omega_{1}=0.03$ and (a) $k=0.029994$, (b) $k=0.029947$, (c) $k=0.02986$, (d) $k=0.02973$.

Similarly, in region b

$$
\begin{aligned}
\phi_{0}^{b} & =-\tanh \left[\left(z-z_{2}\right) \sqrt{\frac{A}{2}}\right] \\
\Gamma_{0}^{b} & =-\sqrt{\frac{2}{9 A}}+\frac{k_{2}}{\tanh ^{3}\left[\left(z-z_{2}\right) \sqrt{A / 2}\right]},
\end{aligned}
$$

where $k_{2}$ is a constant of integration. The solutions in regions a and $\mathrm{b}$ are linked by conditions at $z=z_{1}$, namely, that $d \phi / d z=0$ with $\phi$ and $\Gamma$ continuous. Continuity of $\phi$ requires $z_{1}-z_{0}=z_{2}-z_{1} \equiv Z$, say, so that $z_{1}=\left(z_{0}+z_{2}\right) / 2$. The zero derivative for $\phi$ then implies that $\operatorname{sech}^{2}[Z \sqrt{A / 2}]=o(1)$, so that $Z \rightarrow \infty$ as $\epsilon \rightarrow 0$. Thus the widths of regions a and b become infinite as $\epsilon \rightarrow 0$. Further details of these widths are not determined at leading order, but higher order solutions (omitted for brevity) show that $Z=O_{s}(\log \epsilon)$ as $\epsilon \rightarrow 0$. Finally, continuity of $\Gamma$ at $z=z_{1}$ gives a relationship between $k_{1}$ and $k_{2}$ :

$$
k_{2}=k_{1}+\sqrt{\frac{8}{9 A}} ;
$$

here I use the fact that $\tanh (Z \sqrt{A / 2})=1+o(1)$ as $\epsilon \rightarrow 0$. 


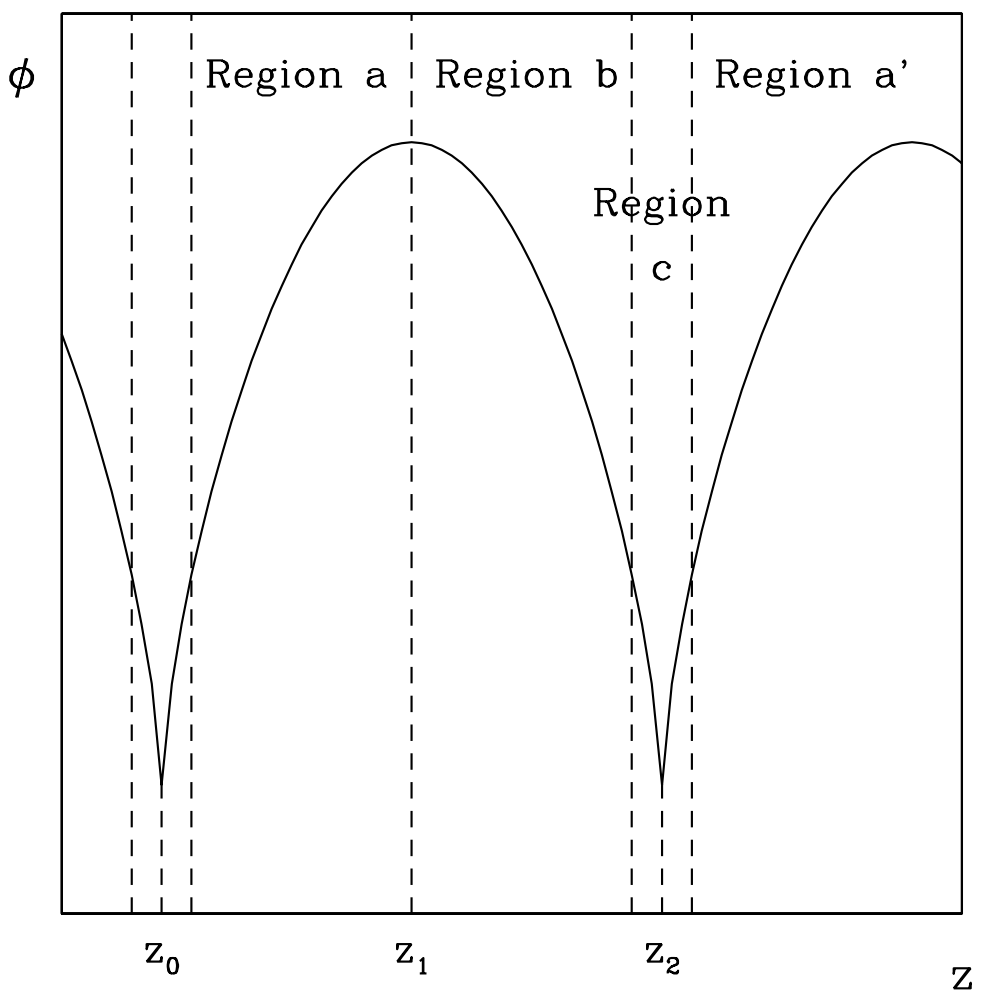

FIG. 7. A schematic illustration of one cycle of the solution for $\phi$ of (3.5) when $\omega_{1}$ is small, illustrating the different regions into which the solution is divided for the perturbation theory calculation.

In region c, which is centered on the minimum of $\phi$, a rescaling of the variables is required. Numerical solutions suggest that $\phi$ is small and $\Gamma$ large in this region, with rapid changes in $z$. Therefore I substitute

$$
\tilde{\phi}=\frac{\phi}{\nu_{1}}, \quad \tilde{\Gamma}=\Gamma \cdot \nu_{2}, \quad \zeta=\frac{z-z_{2}}{\nu_{1}}
$$

into (3.5), where $\nu_{1}$ and $\nu_{2}$ are $o(1)$ as $\epsilon \rightarrow 0$; the rescaling of $\phi$ and $z$ must be the same to allow matching of $\phi$ in regions $\mathrm{b}$ and c. This gives

$$
\begin{aligned}
\frac{d^{2} \tilde{\phi}}{d \zeta^{2}} & =-\nu_{1}^{2} A \tilde{\phi}\left(1-\nu_{1}^{2} \tilde{\phi}^{2}\right)+\epsilon A^{2} \nu_{1}^{4} \tilde{\phi}^{3}\left(\frac{\tilde{\Gamma}^{2}}{\nu_{2}^{2}}-1\right), \\
\frac{d \tilde{\Gamma}}{d \zeta} & =\frac{\nu_{2}-3 \tilde{\Gamma} d \tilde{\phi} / d \zeta-\nu_{1}^{2} \nu_{2} \tilde{\phi}^{2}}{\tilde{\phi}} .
\end{aligned}
$$

Therefore the distinguished limit has $\epsilon^{1 / 2} \nu_{1}^{2} / \nu_{2}=1$, in which case the leading order solutions $\tilde{\phi}_{0}^{c}$ and $\tilde{\Gamma}_{0}^{c}$ satisfy

$$
\begin{aligned}
\frac{d^{2} \tilde{\phi}_{0}^{c}}{d \zeta^{2}} & =A^{2} \tilde{\phi}_{0}^{c 3} \tilde{\Gamma}_{0}^{c 2}, \\
\frac{d \tilde{\Gamma}_{0}^{c}}{d \zeta} & =\frac{-3 \tilde{\Gamma}_{0}^{c} d \tilde{\phi}_{0}^{c} / d \zeta}{\tilde{\phi}_{0}^{c}} .
\end{aligned}
$$


Thus $\tilde{\Gamma}_{0}^{c}=k_{3} / \tilde{\phi}_{0}^{c 3}$ and

$$
\left(\frac{d \tilde{\phi}_{0}^{c}}{d \zeta}\right)^{2}=k_{4}-\frac{A^{2} k_{3}^{2}}{\tilde{\phi}_{0}^{c 2}} .
$$

Here $k_{3}$ and $k_{4}$ are constants of integration, which are determined by matching the solution to that in region b. As $\zeta \rightarrow \pm \infty$, (4.4) implies that $\tilde{\phi}_{0}^{c}= \pm k_{4}^{1 / 2} \zeta+o(\zeta)$, so that $\tilde{\Gamma}_{0}^{c}= \pm k_{3} /\left(k_{4}^{3 / 2} \zeta^{3}\right)+o\left(\zeta^{-3}\right)$. In comparison, as $z \rightarrow z_{2}^{-}, \phi_{0}^{b} \sim-\left(z-z_{2}\right) \sqrt{A / 2}$ and $\Gamma_{0}^{b} \sim(2 / A)^{3 / 2} k_{2}\left(z-z_{2}\right)^{-3}$. Therefore, matching requires $k_{4}=A / 2, k_{2}=k_{3}$, and $\nu_{2}=\nu_{1}^{3} \Rightarrow \nu_{1}=\epsilon^{1 / 2}, \nu_{2}=\epsilon^{3 / 2}$.

The final step in the leading order solution is to determine behavior in region $\mathrm{a}^{\prime}$. The solution here is the same as in region a, but with new constants of integration:

$$
\begin{aligned}
\phi_{0}^{a^{\prime}} & =\tanh \left[\left(z-z_{0}^{\prime}\right) \sqrt{\frac{A}{2}}\right], \\
\Gamma_{0}^{a^{\prime}} & =\sqrt{\frac{2}{9 A}}+\frac{k_{1}^{\prime}}{\tanh ^{3}\left[\left(z-z_{0}\right) \sqrt{A / 2}\right]} .
\end{aligned}
$$

Matching this solution with that in region $\mathrm{c}$ is directly analogous to the matching of solutions in regions b and $\mathrm{c}$, and requires $k_{1}^{\prime}=k_{3}$. Hence $k_{1}^{\prime}=k_{1}+\sqrt{8 / 9 A}$.

Consider now a solution of the form illustrated in Figure 6, and with $N$ local maxima in $\phi$ before $\phi$ approaches 1 asymptotically. Let $k_{1}^{i}$ be the constant of integration $k_{1}$ in the leading order solution in region a before the $i$ th maximum $(i=1, \ldots, N-1)$ or in the part of the solution in which $\phi$ approaches 1 asymptotically $(i=N)$. Then I have shown that $k_{1}^{i}=k_{1}^{i-1}+\sqrt{8 / 9 A}$. Now I require $\Gamma$ finite at $z=0$, and $\Gamma \rightarrow 1$ as $z \rightarrow \infty$. Thus $k_{1}^{1}=0$ and $\sqrt{2 / 9 A}+k_{1}^{N}=1+O\left(\epsilon^{1 / 2}\right)$; the correction is based on the next order term in the expansion for $\Gamma$ in region a (omitted for brevity). Therefore $\sqrt{2 / 9 A}+(N-1) \sqrt{8 / 9 A}=1$, which can be rearranged to give $A=\frac{8}{9}\left(N-\frac{1}{2}\right)^{2}$.

This calculation shows that there are a discrete but infinite set of values of $A$ for which (4.1) has a solution of the required form. These correspond to the values of $k$ plotted in Figure 4. To make this correspondence precise, recall that $A=\alpha / k^{2}$, with $\alpha=1-k / \omega_{1}$ and $\omega_{1}=\epsilon^{1 / 2}$, so that $k=\epsilon^{1 / 2}-A \epsilon^{3 / 2}+O\left(\epsilon^{5 / 2}\right)$. Therefore, at least for sufficiently small $\omega_{1}$, there are solutions of (3.5) for an infinite set of values of $k$, given by $k=\omega_{1}-\frac{8}{9}\left(N-\frac{1}{2}\right)^{2} \omega_{1}^{3}+O\left(\omega_{1}^{5}\right)(N=1,2, \ldots)$. The solution with index $N$ has $N-1$ local maxima and minima in $\phi$. The values of $\phi$ at these extrema depend on $\epsilon$ : the minima have a height that is $O\left(\epsilon^{1 / 2}\right)=O\left(\omega_{1}\right)$, and the leading order correction to $\phi$ in regions a and $\mathrm{b}$ is $O(\epsilon)$, implying that the maxima have a height that is $1-O(\epsilon)=1-O\left(\omega_{1}^{2}\right)$.

5. Solutions for general $\boldsymbol{\omega}_{1}$. In the plot of the critical values of $k$ in Figure 4, I superimpose a plot of the amplitude of the PTW that develops in numerical solutions of (2.1) subject to $u=v=0$ at $x=0$ on $0<x<\infty$. In every case, this corresponds to the critical value of $k$ with largest absolute value. This is despite the fact that each of the other critical values of $k$ corresponds to a possible long term solution of (2.1). In this section, I will discuss in more detail the structure of the $\phi-w-\Gamma$ phase plane, to give further insight into the equation forms at different critical values of $k$.

I begin by investigating the behavior of (3.5) near $\phi=0$, which is a singularity. As $\phi \rightarrow 0$, simple inspection of (3.5c) shows that $|d \Gamma / d z| \rightarrow \infty$ away from the curve $w \Gamma=1 / 3$. Behavior near this curve requires more careful investigation, and I look 
for trajectories of the form $w=w_{0}+\tilde{w}(\phi), \Gamma=w_{0} / 3+\tilde{\Gamma}(\phi)$, where $w_{0}$ is an arbitrary constant and $\tilde{w}$ and $\tilde{\Gamma}$ are $o(1)$ as $\phi \rightarrow 0$. Substituting these solution forms into (3.5) shows that to leading order

$$
\tilde{w}(\phi)=\frac{-\alpha}{2 k^{2} w_{0}} \phi^{2}, \quad \tilde{\Gamma}(\phi)=\frac{1}{10 w_{0}}\left(\frac{\alpha}{w_{0}^{2} k^{2}}-2\right) \phi^{2} .
$$

Therefore, despite the singularity of (3.5) at $\phi=0$, there is a family of nonsingular trajectories which cross the $\phi=0$ plane through the curve $\Gamma w=1 / 3$. Such a curve is sometimes known as a "hole in a singular barrier" (Perumpanani et al. (1999); Pettet, McElwain, and Norbury (2000)). Of the trajectories crossing $\phi=0$ through this curve, only those crossing at positive values of $w$ and $\Gamma$ are of interest, and taken together, these make up a surface in $\phi-w-\Gamma$ phase space, which I denote by $\mathcal{S}(k)$. There is a trajectory of the required form for a given value of $k$ if and only if this surface $\mathcal{S}(k)$ contains the point $\phi=1, w=0, \Gamma=1$.

The surface $\mathcal{S}(k)$ has a very complex form, especially for small values of $k$, making visualization in three dimensions very difficult. I have found it most instructive to plot a cross section of the surface, and a natural cross section is the $w=0$ plane, which is illustrated in Figure 8 for the largest three critical values of $k$ when $\omega_{1}=1$. Note that, in each case, the intersection includes the point $\Gamma=\phi=1$.

In Figure 8(a) the trajectories making up $\mathcal{S}(k)$ intersect the $w_{z}<0$ portion of the $w=0$ plane only once. This implies that the corresponding solution of (3.5), (3.6) is monotonic in $\phi$, and numerical results suggest that this is also true for other $\omega_{1}$, when $k$ is at its largest critical value. Conversely, for the other critical values of $k$, numerical solutions suggest that the trajectory passing through $\phi=\Gamma=1, w=0$ does so only after previously crossing the $w=0$ plane. Based on this, I hypothesize that for given $\omega_{1}$ there is only one solution of (3.5), (3.6) that is monotonic in $\phi$, namely, that corresponding to the largest initial value of $k$. Further, I hypothesize that any solutions of (3.5) with nonmonotonic $\phi$ are unstable as solutions of (2.1). A number of results of the form "nonmonotonicity implies instability" are known for scalar reaction-diffusion equations (Hagan (1981b), Henry (1981)), and numerical simulations using the solutions of (3.5), (3.6) with small perturbations as initial conditions for (2.1) suggest that a corresponding result applies in this case. Taken together, these hypotheses provide an explanation for the solution of (2.1) always corresponding to the solution of (3.5), (3.6) with the largest critical value of $k$.

Although I cannot prove these hypotheses, I will present a sketch proof of the first one. Proof that a solution that is monotonic in $\phi$ exists for some value of $k$ is obtained by direct construction. Numerical solutions of (2.1) with $u=v=0$ at $x=0$ suggest that the ratio of $\Psi$ and $R$ is constant in the observed solution. Based on this, I look for a solution of (3.5) in which $\Gamma \equiv 1$. The equations (3.5) then give

$$
\begin{aligned}
\phi_{z} & =w, \\
w_{z} & =-\left(\frac{\alpha}{k^{2}}\right) \phi\left(1-\phi^{2}\right), \\
3 w+\phi^{2} & =1 .
\end{aligned}
$$

Combining (5.2a) and (5.2b), and requiring $\phi=1$ when $w=0$, gives

$$
w^{2}=\frac{\alpha}{2 k^{2}}\left(1-\phi^{2}\right)^{2} .
$$

This is consistent with (5.2c) if and only if $k^{2}=9 \alpha / 2$. (Recall that the sign of $k$ must 


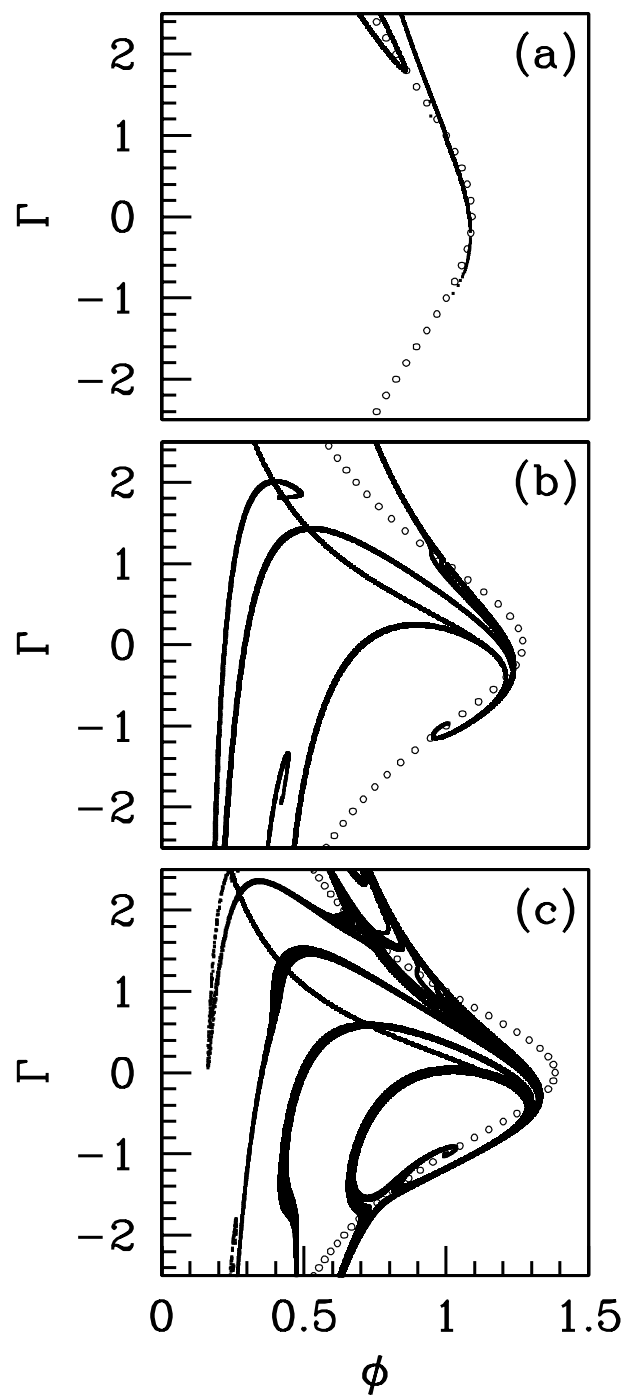

FIG. 8. An illustration of the intersection between the surface $\mathcal{S}(k)$ and the $w=0$ plane for the largest three critical values of $k$ when $\omega_{1}=1$. The small circles represent the curve $\phi^{2}\left[1-\alpha+\alpha \Gamma^{2}\right]=1$; to the right of this curve, $w_{z}>0$, so that the trajectories are crossing from negative to positive $w$, and to the left of the curve, $w_{z}<0$. I have solved (3.5) numerically for values of $w_{0}$ increasing from 0.01 in increments of $10^{-6}$; initial conditions are $w=w_{0}+\tilde{w}$, $\Gamma=\Gamma_{0}+\tilde{\Gamma}$ with $\tilde{w}$ and $\tilde{\Gamma}$ given by (5.1), and $\phi=0.001$. In each of these solutions, I record and plot each point at which the $w=0$ plane is crossed. The values of $k$ are (a) $k=0.842$, (b) $k=0.621$, (c) $k=0.523$.

be the same as that of $\omega_{1}$.) Recalling that $\alpha=1-k / \omega_{1}$, this implies

$$
k=k^{*} \equiv \frac{-9+\sqrt{81+72 \omega_{1}^{2}}}{4 \omega_{1}} .
$$

Note that the solution trajectory corresponding to this value of $k$ is monotonic in $w$ as well as $\phi$, and thus lies within $\hat{\mathcal{S}}(k)$, the subset of $\mathcal{S}(k)$ formed by the portion of the trajectories starting on $\phi=0, w \Gamma=1 / 3$, until they leave the region $\phi>0, w>0$, $\phi^{2}\left[1+\alpha\left(\Gamma^{2}-1\right)\right]<1$; this last condition is simply $w_{z}<0$. 
To study uniqueness, it is convenient to consider varying $k$ with $\alpha$, rather than $\omega_{1}$, fixed. Suppose that there is a value of $k, k_{1}$ say, not equal to $k^{*}$, for which there is a trajectory that is monotonic in $\phi$ and connects $\phi=0, w \Gamma=1 / 3$ with $w=0$, $\phi=\Gamma=1$. To be specific, I assume that $k_{1}<k^{*}$, though a corresponding argument is valid if $k_{1}>k^{*}$. The solution trajectories for $k=k_{1}$ and $k^{*}$ are contained in $\mathcal{S}\left(k_{1}\right)$ and $\hat{\mathcal{S}}\left(k^{*}\right)$, respectively; note that in general the trajectories for $k^{*}$ and $k_{1}$ will cross $\phi=0$ at different points along $w \Gamma=1 / 3$. Straightforward examination of the eigenvalues and eigenvectors of $(3.5)$ at $w=0, \phi=\Gamma=1$ implies that $\mathcal{S}\left(k_{1}\right)$ lies above $\hat{\mathcal{S}}\left(k^{*}\right)$ close to this point, in the sense that $w$ is greater on $\mathcal{S}\left(k_{1}\right)$ than on $\hat{\mathcal{S}}\left(k^{*}\right)$. Moreover eliminating $w_{0}$ in (5.1) implies that $\hat{\mathcal{S}}\left(k^{*}\right)$ lies above $\mathcal{S}\left(k_{1}\right)$ for sufficiently small $\phi$. This suggests that the surfaces $\mathcal{S}\left(k_{1}\right)$ and $\hat{\mathcal{S}}\left(k^{*}\right)$ intersect, which is impossible since $(3.5 \mathrm{~b})$ implies that $w_{z}$ increases with $k$ in the region $\phi^{2}\left[1+\alpha\left(\Gamma^{2}-1\right)\right]<1$. It remains possible that $\mathcal{S}\left(k_{1}\right)$ and $\hat{\mathcal{S}}\left(k^{*}\right)$ wind around one another; I have been unable to rule this out, although numerical solutions suggest that it does not happen.

6. The form of the observed periodic travelling wave. The sketch proof in the previous section is constructive, in the sense that it explicitly determines a formula for $k^{*}$. The corresponding solution of (3.5) can easily be determined from (5.2c) as $\phi=\tanh (z / 3)$. Converting back to the original variables gives

$$
R(x)=r_{p t w} \tanh \left(\frac{x}{\sqrt{2}}\right), \quad \Psi(x)=\psi_{p t w} \tanh \left(\frac{x}{\sqrt{2}}\right)
$$

where

$$
r_{p t w}=\left\{\frac{1}{2}\left[1+\sqrt{1+\frac{8}{9} \omega_{1}^{2}}\right]\right\}^{-1 / 2}, \quad \psi_{p t w}=-\operatorname{sign}\left(\omega_{1}\right)\left\{\frac{\sqrt{1+\frac{8}{9} \omega_{1}^{2}}-1}{\sqrt{1+\frac{8}{9} \omega_{1}^{2}}+1}\right\}^{1 / 2}
$$

The solution given by (6.1), (6.2) is an excellent match with the long term behavior predicted by numerical simulations of (2.1) subject to $u=v=0$ at $x=0$, as illustrated in Figure 4. Moreover, (6.2) enables direct determination of the properties of PTWs generated by Dirichlet boundary conditions. For example, substitution of (6.2) into (2.3) gives the condition for PTW stability as

$$
8 \omega_{1}^{6}+16 \omega_{1}^{4}-10 \omega_{1}^{2}-27<0 \Longleftrightarrow\left|\omega_{1}\right|<1.110468 \ldots .
$$

A detailed numerical study shows that cases such as that shown in Figure 3, in which irregular oscillations develop, correspond exactly to values of $\omega_{1}$ above this critical value. Similarly the direction of the PTWs can be determined - this depends on $\omega_{0}$ as well as $\omega_{1}$. The PTW solution for $u$ and $v$, given in (2.2), moves in the positive $x$-direction if and only if $\left(\omega_{0}-\omega_{1} r^{2}\right)$ and $\theta_{x}$ have opposite signs. Therefore the PTW given by (6.2) moves in the positive $x$-direction if and only if

$$
\begin{aligned}
\psi_{p t w} \cdot\left(\omega_{0}-\omega_{1} r_{p t w}^{2}\right)<0 \Leftrightarrow & \omega_{0} \text { and } \omega_{1} \text { have the same sign } \\
& \text { and }\left|\omega_{0}\right|>\frac{2\left|\omega_{1}\right|}{1+\sqrt{1+\frac{8}{9} \omega_{1}^{2}}} .
\end{aligned}
$$

Conditions (6.3) and (6.4) are illustrated graphically in Figure 9. 


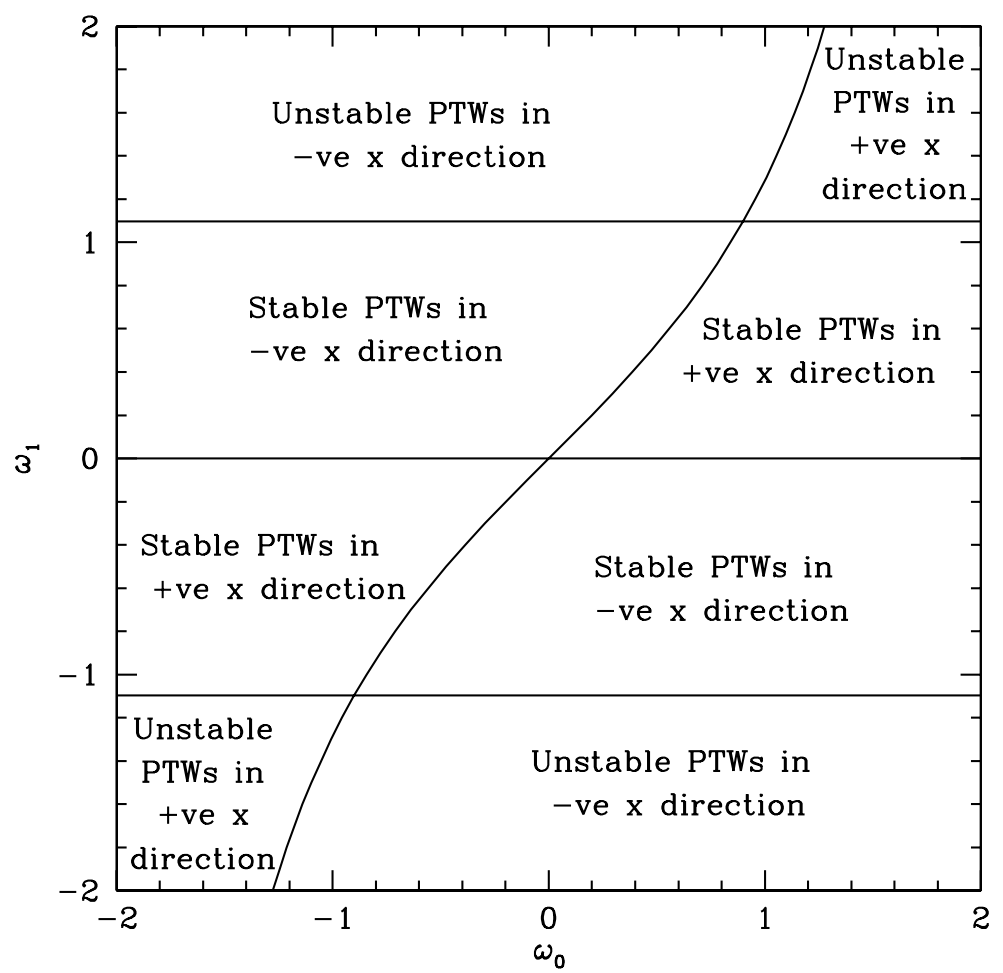

FIG. 9. An illustration of conditions (6.3) and (6.4) for the stability and direction of the PTW solution generated in the region $x>0$ by the boundary condition $u=v=0$ at $x=0$.

7. Discussion. Using a combination of analysis and numerical simulation, I have shown that when the $\lambda-\omega$ system (2.1) is solved on a finite domain subject to zero Dirichlet boundary conditions, PTWs develop. I have shown that there is a discrete family of possible wave amplitudes for which solutions exist, but my results suggest that in only one of these cases does the amplitude vary monotonically in space. I hypothesize that this family is the only stable solution, implying that the boundary conditions select a unique PTW amplitude that is independent of initial conditions. A formula for this amplitude is given in (6.2).

An obvious extension of the work presented in this paper is to consider behavior in two spatial dimensions. I have done a limited program of numerical simulations of the $\lambda$ - $\omega$ system (2.1) in two dimensions, and a typical result is illustrated in Figure 10. For this figure, equations (2.1) were solved on an approximately circular but irregular domain, with the boundary condition $u=v=0$. A solution of "target pattern" form develops, moving inwards from the boundary; an animation of this solution can be seen at www.ma.hw.ac.uk/ jas/supplements/dirichlet/. This solution is a natural two-dimensional extension of the one-dimensional results I have been discussing, in which planar PTW solutions are modulated by the curvature of the domain. A natural topic for future analytical work is the spatial scale over which the curvature of the wave fronts varies.

The mathematical study of PTWs has been given a significant boost recently by the identification by ecologists of PTWs in cyclic populations. This empirical work is slow, requiring spatiotemporal field data gathered over many years. (Pop- 


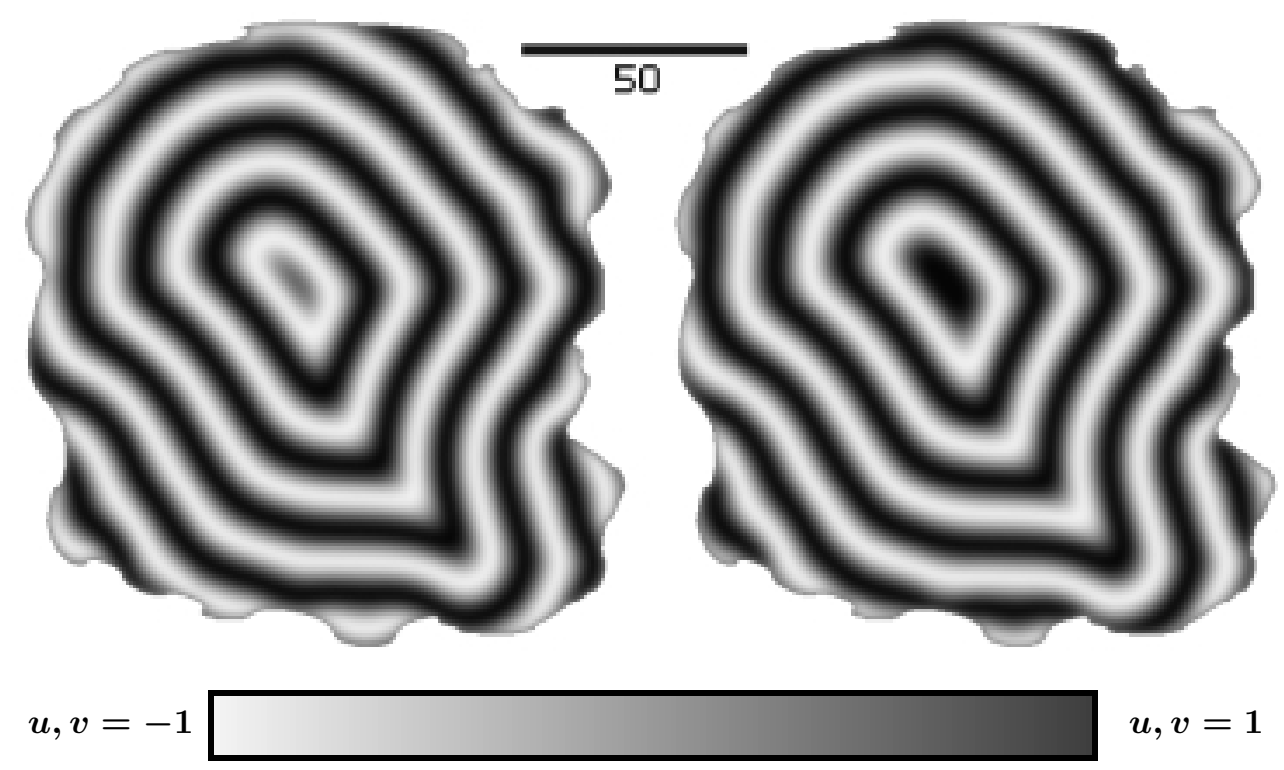

FIG. 10. Numerical simulation of the $\lambda-\omega$ system (2.1) in two space dimensions on an irregular domain with boundary condition $u=v=0$ on the perimeter. The solutions for $u$ (left) and $v$ (right) are shown at time $t=400$; a "target pattern"-type solution moves inwards from the boundary towards the center of the domain. The initial conditions (at $t=0)$ are generated randomly, and the parameters are $\omega_{0}=\omega_{1}=1$. The size of the spatial domain is indicated by the scale bar, which is 50 space units long. An animation corresponding to this figure can be seen at http://www.ma.hw.ac.uk/ jas/supplements/dirichlet/. The equations were solved numerically using an alternating direction semi-implicit method.

ulation cycles typically have a period of between 4 and 10 years.) The analysis of the data then depends on spatiotemporal statistical methods developed only recently (Bjørnstad, Ims, and Lambin (1999)). For these reasons, it is too early to assess how widespread PTWs are in real populations. However, there is now very strong evidence for the existence of a PTW in cyclic field vole populations in the Kielder forest, on the Scotland-England border (Lambin et al. (1998); MacKinnon et al. (2001)), and more limited evidence for PTWs in various other populations, including red grouse in Northeast Scotland (Moss, Elston, and Watson (2000)), water voles in Eastern France (Giraudoux et al. (1997)), and larch budmoths in the European Alps (Bjørnstad et al. (2002)). The major question raised by these ecological studies is, what are the cause(s) of the PTWs? One possibility is that the PTWs are generated by the invasion of a prey population by predators (Sherratt, Lewis, and Fowler (1995); Sherratt et al. (2000)). However, once a whole domain has been invaded, PDE models predict that the waves will gradually die out (with zero flux boundary conditions; see Kay and Sherratt (1999)). Thus this mechanism requires a recent invasion, which does not apply in most cases. In contrast, the mechanism studied in this paper is consistent with conditions found in many real ecological systems. A Dirichlet boundary condition (with population density equal to zero) is appropriate when the domain is surrounded by a region of different habitat in which the population cannot survive - for example, an area of woodland surrounded by open fields. In a companion paper (Sherratt et al. (2002)), coworkers and I show that numerical simulations of both PDE and spatially discrete predator-prey models predict the generation of periodic waves by Dirichlet 
boundary conditions. Extension of the analytical results in the present paper to realistic predator-prey models is a major challenge for future work, which would enable systematic prediction of the occurrence of periodic waves in real populations.

Acknowledgments. This work arose from discussions with Tom Sherratt and Xavier Lambin, to whom I am very grateful. In particular, the original idea for periodic wave generation by Dirichlet boundary conditions in ecological systems is due to Tom Sherratt. I also thank Vadim Biktashev and Jack Carr for helpful discussions.

\section{REFERENCES}

P. Ashwin, M. V. Bartuccelli, T. J. Bridges, and S. A. Gourley (2002), Travelling fronts for the KPP equation with spatio-temporal delay, Z. Angew. Math. Phys., 53, pp. 103-122.

J. F. G. Auchmuty AND G. Nicolis (1976), Bifurcation analysis of reaction-diffusion equations. III. Chemical oscillations, Bull. Math. Biol., 38, pp. 325-350.

O. N. Buørnstad, R. A. Ims, And X. Lambin (1999), Spatial population dynamics: Analyzing patterns and processes of population synchrony, Trends in Ecology and Evolution, 14, pp. 427432.

O. N. Buørnstad, M. Peltonen, A. M. Liebhold, W. Baltensweiler (2002), Waves of larch budmoth outbreaks in the European Alps, Science, 298, pp. 1020-1023.

G. B. Ermentrout (1981), Stable small amplitude solutions in reaction-diffusion systems, Quart. Appl. Math., 39, pp. 61-86.

G. B. Ermentrout, X. Chen, And Z. Chen (1997), Transition fronts and localised structures in bistable reaction-diffusion equations, Phys. D, 108, pp. 147-167.

G. B. Ermentrout and N. Kopell (1984), Frequency plateaus in a chain of weakly coupled oscillators, I, SIAM J. Math. Anal., 15, pp. 215-237.

G. B. Ermentrout and N. Kopell (1986), Symmetry and phase-locking in chains of weakly coupled oscillators, Commun. Pure Appl. Anal., 49, pp. 623-660.

P. Giraudoux, P. Delattre, M. Habert, J. P. Quere, S. Deblay, R. Defaut, R. Duhamel, M. F. Moissenet, D. Salvi, And D. Truchetet (1997), Population dynamics of fossorial water vole (Arvicola terrestris scherman): A land use and landscape perspective, Agr. Ecosyst. Environ., 66, pp. 47-60.

P. S. Hagan (1981a), Target patterns in reaction-diffusion systems, Adv. Appl. Math., 2, pp. 400416.

P. S. HAGAN (1981b), The instability of non-monotonic wave solutions of parabolic equations, Stud. Appl. Math., 64, pp. 57-88.

D. Henry (1981), Geometric Theory of Semilinear Parabolic Equations, Springer-Verlag, Berlin.

T. KaPitula (1994), On the nonlinear stability of plane waves for the Ginzburg-Landau equation, Comm. Pure Appl. Math., 47, pp. 831-841.

A. L. Kay And J. A. Sherratt (1999), On the persistence of spatiotemporal oscillations generated by invasion, IMA J. Appl. Math., 63, pp. 199-216.

A. L. Kay and J. A. Sherratt (2000), Spatial noise stabilizes periodic wave patterns in oscillatory systems on finite domains, SIAM J. Appl. Math., 61, pp. 1013-1041.

N. KOPELL (1981), Target pattern solutions to reaction-diffusion equations in the presence of impurities, Adv. Appl. Math., 2, pp. 389-399.

N. Kopell And L. N. Howard (1973), Plane wave solutions to reaction-diffusion equations, Stud. Appl. Math., 52, pp. 291-328.

N. KOPELl AND L. N. HowARD (1981), Target patterns and horseshoes from a perturbed central force problem: Some temporally periodic solutions to reaction-diffusion problems, Stud. Appl. Math., 64, pp. 1-56.

N. Kopell, G. B. Ermentrout, and T. L. Williams (1991), On chains of oscillators forced at one end, SIAM J. Appl. Math., 51, pp. 1397-1417.

X. Lambin, D. A. Elston, S. J. Petty, And J. L. MacKinnon (1998), Spatial asynchrony and periodic travelling waves in cyclic populations of field voles, Proc. Roy. Soc. London Ser. B, 265, pp. 1491-1496.

J. L. Mackinnon, X. Lambin, D. A. Elston, C. J. Thomas, T. N. Sherratt, and S. J. Petty (2001), Scale invariant spatio-temporal patterns of field vole density, J. Animal Ecology, 70, pp. 101-111.

K. Maginu (1981), Stability of periodic travelling wave solutions with large spatial periods in reaction-diffusion systems, J. Differential Equations, 39, pp. 73-99. 
R. Moss, D. A. Elston, And A. Watson (2000), Spatial asynchrony and demographic travelling waves during red grouse population cycles, Ecology, 81, pp. 981-989.

H. Nagashima (1991), Target patterns and pacemakers in a reaction-diffusion system, J. Phys. Soc. Japan, 60, pp. 2797-2799.

A. J. Perumpanani, J. Norbury, J. A. Sherratt, and H. M. Byrne (1999), A two parameter family of travelling waves with a singular barrier arising from the modelling of matrix mediated malignant invasion, Phys. D, 126, pp. 145-159.

S. V. Petrovski and H. Malchow (1999), A minimal model of pattern formation in a prey predator system, Math. Comput. Modelling, 29, pp. 49-63.

S. V. Petrovskil And H. Malchow (2000), Critical phenomena in plankton communities: KISS model revisited, Nonlinear Anal. Real World Appl., 1, pp. 37-51.

G. J. Pettet, D. L. McElwain, And J. Norbury (2000), Lotka-Volterra equations with chemotaxis: Walls, barriers and travelling waves, IMA J. Math. Appl. Med. Biol., 17, pp. 395-413.

L. Ren And G. B. Ermentrout (1998), Monotonicity of phaselocked solutions in chains and arrays of nearest-neighbor coupled oscillators, SIAM J. Math. Anal., 29, pp. 208-234.

J. L. Romero, M. L. Gandarias, And E. Medina (2000), Symmetries, periodic plane waves and blow-up of lambda-omega systems, Phys. D, 147, pp. 259-272.

S. K. Scott, B. R. Johnson, A. F. Taylor, and M. R. Tinsley (2000), Complex chemical reactions - A review, Chem. Eng. Sci., 55, pp. 209-215.

J. A. Sherratt (1994a), On the evolution of periodic plane waves in reaction-diffusion systems of $\lambda-\omega$ type, SIAM J. Appl. Math., 54, pp. 1374-1385.

J. A. Sherratt (1994b), Irregular wakes in reaction-diffusion waves, Phys. D, 70, pp. 370-384.

J. A. Sherratt, M. A. Lewis, And A. C. Fowler (1995), Ecological chaos in the wake of invasion, Proc. Natl. Acad. Sci. USA, 92, pp. 2524-2528.

J. A. Sherratt, X. Lambin, C. J. Thomas, and T. N. Sherratt (2002), Generation of periodic waves by landscape features in cyclic predator-prey systems, Proc. Roy. Soc. London Ser. B, 269, pp. 327-334.

T. N. Sherratt, X. Lambin, S. J. Petty, J. L. Mackinnon, C. F. Coles, and C. J. Thomas (2000), Application of coupled oscillator models to understand extensive synchrony domains and travelling waves in populations of the field vole in Kielder forest, UK, J. Appl. Ecol., 37, pp. $148-158$.

J. SNeyd and J. Sherratt (1997), On the propagation of calcium waves in an inhomogeneous medium, SIAM J. Appl. Math., 57, pp. 73-94.

A. T. Winfree (2001), The Geometry of Biological Time, Springer-Verlag, New York. 